\title{
Altered synaptic plasticity in the mossy fibre pathway of transgenic mice expressing mutant amyloid precursor protein
}

\author{
Jonathan Witton ${ }^{1,2}$, Jon T Brown ${ }^{1,2}$, Matthew W Jones ${ }^{1}$, Andrew D Randall ${ }^{1,2^{*}}$
}

\begin{abstract}
$A \beta$ peptides derived from the cleavage of amyloid precursor protein are widely believed to play an important role in the pathophysiology of Alzheimer's disease. A common way to study the impact of these molecules on CNS function is to compare the physiology of transgenic mice that overproduce $A \beta$ with non-transgenic animals. In the hippocampus, this approach has been frequently applied to the investigation of synaptic transmission and plasticity in the perforant and Schaffer collateral commissural pathways, the first and third components of the classical hippocampal trisynaptic circuit, respectively. Similar studies however have not been carried out on the remaining component of the trisynaptic circuit, the mossy fibre pathway. Using transverse hippocampal slices prepared from $\sim 2$ year old animals we have compared mossy fibre synaptic function in wild-type mice and their Tg2576 littermates which age-dependently overproduce A $\beta$. Input-output curves were not altered in slices from Tg2576 mice, but these animals exhibited a significant loss of the prominent frequency-facilitation expressed by the mossy fibre pathway. In addition to this change in short term synaptic plasticity, high frequency stimulation-induced, NMDAreceptor-independent LTP was absent in slices from the transgenic mice. These data represent the first description of functional deficits in the mossy fibre pathway of $A \beta$-overproducing transgenic mice.
\end{abstract}

\section{Background}

The hippocampus is a brain structure with a crucial role in mammalian learning and memory. In transverse section the hippocampus has an iconic morphology around which a trisynaptic loop of glutamatergic pathways navigates. The three sequential glutamatergic pathways that make up this circuit are 1) the perforant path (PP) inputs to the granule cells of the dentate gyrus (DG-GC), 2) the mossy fibre projection (MFP) from DG-GCs to CA3 pyramidal cells (CA3-PC) and, 3) the Schaffer collateral commissural pathway (SCCP) that links CA3-PCs with CA1 pyramidal cells (CA1-PC).

There are a large number of neurological and psychiatric diseases in which measurable cognitive deficits contribute to the disease phenotype. Of these Alzheimer's disease (AD) is perhaps the best known. The prevalence of this condition means that most adults in the developed world will have personal experience from friends

\footnotetext{
* Correspondence: a.d.randall@bristol.ac.uk

'School of Physiology and Pharmacology, University of Bristol, University Walk, Bristol, BS8 1TD, UK

Full list of author information is available at the end of the article
}

or family members of the devastating effects on cognitive function $\mathrm{AD}$ produces in those afflicted with the disease.

The majority of $\mathrm{AD}$ investigators would accept that $A \beta$ peptides play a major role in the pathophysiology of $\mathrm{AD}[1,2]$. These molecules are derived from proteolytic cleavage of amyloid precursor protein (APP) and APP mutations that favour $\mathrm{A} \beta$ generation or $\mathrm{A} \beta$ aggregation lead to familial forms of AD [3]. This has lead to an extensive, and ever-growing, literature on the biological actions of $A \beta$ peptides. Large parts of this body of research involve two major experimental approaches. The first revolves around determining the effects produced by exogenous $A \beta$ peptide preparations introduced acutely (or subacutely) into assay systems of interest. The second approach involves comparing the physiology of wild-type (WT) mice with examples of genetically engineered mouse lines that express transgenes which result in age-dependent over-production of $A \beta$ [4]. There are now very large numbers $(>50)$ of such transgenic lines. The transgenes they express are usually APP variants that promote $A \beta$-production and/or 
aggregation, sometimes combined with other genes involved in the APP-processing pathway, most notably mutant forms of presenilin1 (PS1).

Numerous studies have considered how $A \beta$ affects basal synaptic function and synaptic plasticity in the hippocampus [5]. These investigations have involved exogenous $A \beta$ preparations of various forms as well as $A \beta$-overproducing transgenic animals. Interestingly, despite the large number of such studies performed in recent years, the effects of $A \beta$ peptides have been only been investigated in two of the three pathways of the classic trisynaptic loop [5]. The pathways that have already been studied in depth, the PP and SCCP, are notably those that express NMDA-receptor dependent long-term potentiation (LTP). In contrast, the effects of $\mathrm{A} \beta$ on the MFP from DG granule cells to CA3 neurones, has received little if any attention.

As well as exhibiting presynaptically-expressed NMDA receptor-independent LTP, the MFP has a quite different physiology, anatomy and pharmacology to that of either the PP or SCCP [6-10]. Working in tandem with direct PP inputs to area CA3 the MFP is proposed to play a discrete role in hippocampal information processing and thus, by extension, hippocampally-mediated mnenomic function [11-14]. Here we present a first investigation of synaptic function in the MFP using hippocampal slices prepared from the commonly studied A $\beta$-over-expressing mouse line, Tg2576. When compared to WT littermates, we found both short and long term synaptic plasticity were altered in this transgenic line.

\section{Methods}

The animals used for this study were 24-25 month old male Tg2576 mice and their WT littermates [15]. At this advanced age Tg2576 mice have both elevated A $\beta$ and amyloid plaque pathology. Tg2576 mice express human APP harbouring the Swedish mutation (APPSwe) under control of the hamster prion protein promoter, which is expressed highly in the brain (but is not neuronal specific). The mice were on a mixed genetic background of $\mathrm{C} 57 \mathrm{Bl} / 6 \mathrm{SJL}$ and $\mathrm{C} 57 \mathrm{Bl} / 6$. After weaning, selection of male pups and PCR-based genotyping the mice were singly housed on a 12:12 hr light:dark cycle with ad lib access to food and water.

Following sacrifice by cervical dislocation the hippocampus was dissected free from the rest of the brain and $400 \mu \mathrm{m}$ thick transverse hippocampal slices were prepared using methods similar to those of others e.g. [16]. Following a post-slicing recovery period of $>1 \mathrm{hr}$ slices were transferred to an interface-type recording chamber constantly perfused with artificial cerebrospinal fluid (aCSF) at $33^{\circ} \mathrm{C}$. The composition of the aCSF was $(\mathrm{mM}) \mathrm{NaCl}, 124 ; \mathrm{KCl}, 3 ; \mathrm{NaHCO}_{3}, 26 ; \mathrm{CaCl}_{2}, 2$;
$\mathrm{NaH}_{2} \mathrm{PO}_{4}, 1.25 ; \mathrm{MgSO}_{4}, 1$; D-glucose, 10. This aCSF was equilibrated with $95 \% \mathrm{O}_{2}$ and $5 \% \mathrm{CO}_{2}$ by constant bubbling.

Extracellular recordings where made from stratum lucidum using aCSF filled glass electrodes of $~ 3 \mathrm{MOhm}$ resistance. Field excitatory postsynaptic potentials (fEPSPs) were evoked in response to brief electrical stimuli applied via a concentric bipolar stimulating electrode placed deep in the hilar region close to the dentate granule cell layer. Responses were amplified $(1000 \times)$, lowpass filtered at $10 \mathrm{kHz}$ and digitally sampled to a personal computer at $50 \mathrm{kHz}$ using pClamp10 software. All experiments were performed in the presence of L-689,560 ( $5 \mu \mathrm{M}$, Tocris) to entirely block NMDA receptor function. The experimenter was blind to genotype during the recordings.

Initially, input-output curves were collected by varying the stimulation strength applied in the range 50-300 $\mu \mathrm{A}$, in $25 \mu \mathrm{A}$ intervals. For subsequent investigations of synaptic plasticity the stimulus strength was set to a level that produced $\sim 50 \%$ of the maximal response. As suggested by the pooled input-output data from the two genotypes (see Figure 1A), the stimulus intensity required to do this was not different in the two groups (WT $112.5 \pm 4.7 \mu \mathrm{A}, \operatorname{Tg} 2576112.5 \pm 9.4 \mu \mathrm{A}$ ).

Short-term synaptic plasticity was investigated in two ways. Firstly, we collected paired-pulse profiles with inter-stimulus intervals varying logarithmically over two orders of magnitude (10-1000 ms). Secondly, the increase in synaptic response seen during a 20 pulse, 1 $\mathrm{Hz}$ stimulus train was determined. To study LTP, we recorded baseline synaptic transmission in response stimuli applied every 30s for at least 10 minutes. We then attempted to induce NMDA receptor-independent LTP with a conditioning stimulus consisting of $1 \mathrm{~s}, 100 \mathrm{~Hz}$ stimulus train, repeated three times with an inter-train interval of $10 \mathrm{~s}$. The responses to stimuli applied every 30 s were then followed for another 30 minutes, at which time we determined the synaptic depression produced by application of DCG-IV $(2 \mu \mathrm{M})$. Depression greater than $75 \%$ by this group II mGluR agonist was used to confirm we had been studying a largely pure MFP input [17].

Data were analysed using a combination of pClamp software and custom-written code within the MATLAB numerical processing environment. For LTP experiments amplitude measurements were made on averages of 4 consecutive EPSPs, each of which therefore cover a 2 minute time window. Short-term plasticity protocols were typically run three times on each slice and responses averaged. Input-output curves were compared with a 2-way ANOVA. Paired pulse facilitation at $1 \mathrm{~s}$ was analysed using Student's t-test. This test was also used to compare the area under the frequency 


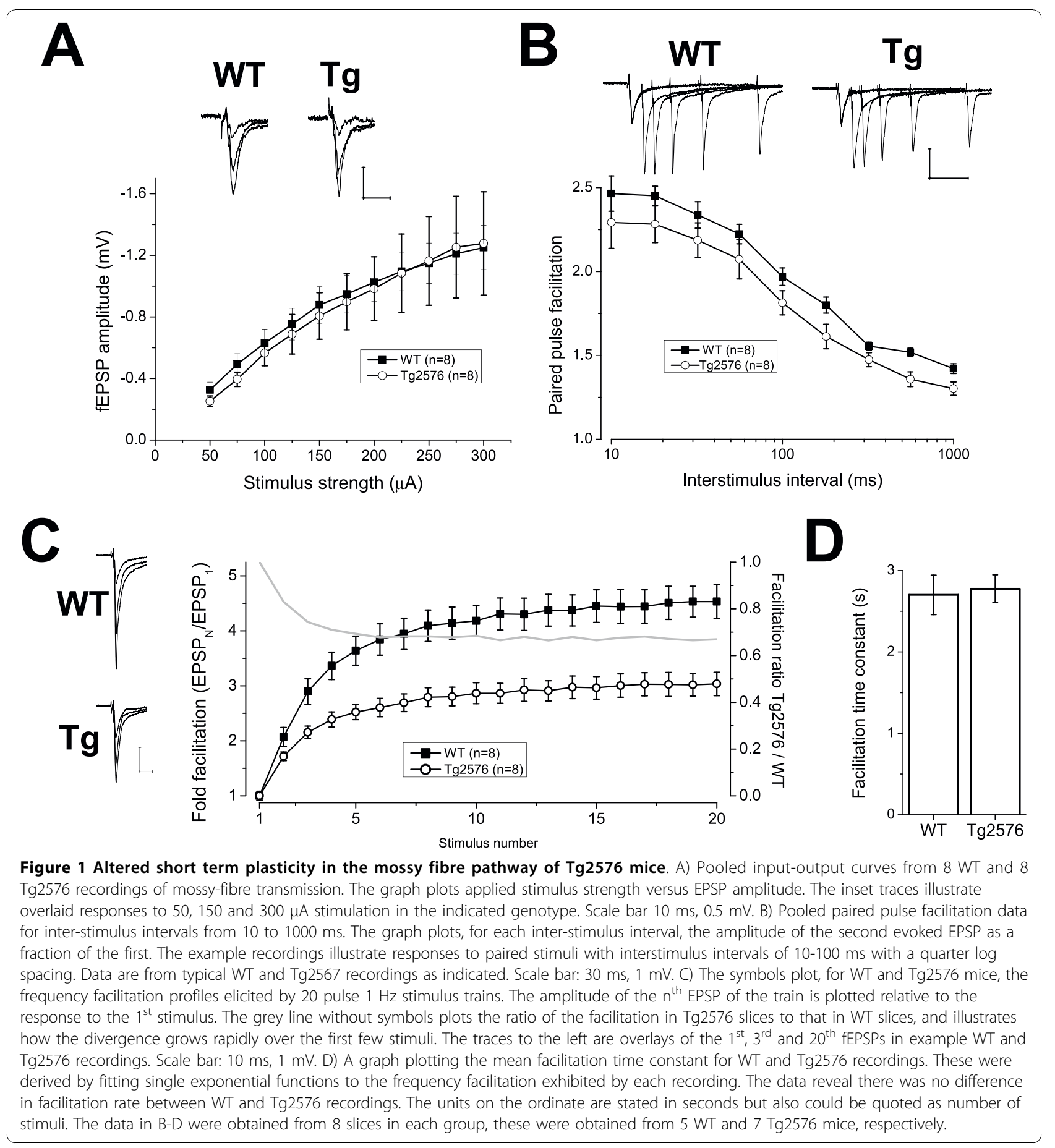

facilitation curves. Comparisons of the extent of LTP in WT and Tg2576 mice were based on a Student's t-test performed on the mean of the last 2 minutes of synaptic responses recorded before DCG-IV application.

\section{Results}

MFP-mediated synaptic responses with classical fast negative-going waveforms and DCG-IV-sensitivity were present in hippocampal slices prepared from both WT and Tg2576 mice. Input-output curves were compiled with stimulus strengths between 50-300 $\mu \mathrm{A}$. These exhibited the expected increase in EPSP amplitude as stimulus strength was increased. Although the relationship between EPSP amplitude and stimulus strength was, not unexpectedly, quite variable from slice to slice, pooled input-output curves from WT and Tg2576 mice 
were very similar (Figure $1 \mathrm{~A}, \mathrm{P}>0.85$ ). Thus, there does not seem to be a profound alteration to basal MF synaptic transmission in the $A \beta$-overproducing mice, or at least one that can be resolved with the extracellular recording methodologies employed here.

A hallmark of MF inputs to CA3-PCs in young animals is a pronounced short-term synaptic plasticity. This is evident in both substantial paired pulse facilitation (PPF) and frequency facilitation (FF) elicited by trains of stimuli. MFP responses in slices from both aged WT and Tg2576 mice exhibited marked PPF (Figure 1B); however, at all inter-stimulus latencies PPF in the Tg2576 group $(\mathrm{n}=8)$ was between 5 and $10 \%$ less that than that in the WT group $(\mathrm{n}=8)$, indicating that short term synaptic plasticity might be disturbed in the $A \beta$-overproducing mice. At the longest inter-stimulus interval tested, $1 \mathrm{~s}$, PPF in Tg2576 slices was significantly smaller $(91.6 \pm 2.7 \%)$ than that in slices from WT mice $(\mathrm{P}<0.03$, unpaired $\mathrm{t}$-test). When stimulation was repeatedly applied at this same inter-stimulus interval (i.e. $1 \mathrm{~Hz}$ ) 20 times in succession marked frequency facilitation developed. Although facilitation occurred in both groups it was substantially attenuated in slices from Tg2576 mice (Figure 1C). The area under these facilitation curves was $57.4 \pm 2.8$ in WT slices and 33.3 \pm 3.3 in $\mathrm{Tg} 2576$ mice, a highly significant difference $(\mathrm{P}<0.00008$, unpaired $\mathrm{t}$-test $)$. The rates with which frequency facilitation developed during these $1 \mathrm{~Hz}$ trains were not different, with time constants of $2.70 \pm 0.24 \mathrm{~s}$ and $2.78 \pm 0.17 \mathrm{~s}$ in WT and Tg2576 mice, respectively $(\mathrm{P}<0.88$, Figure 1D). As the number of stimuli delivered was increased, the ratio of the facilitation observed in WT and Tg2576 animals fell to a plateau of $\sim 0.67$ over the first $\sim 6$ stimuli (Figure $1 \mathrm{C}$, grey line); this difference between the two groups developed exponentially with a rate of 0.76 stimuli $^{-1}$.

To investigate MF-LTP in these animals, we first recorded a period of stable baseline synaptic transmission in response to stimuli applied every $30 \mathrm{~s}$. Following 10 or more minutes of stable EPSP amplitude we applied three $1 \mathrm{~s}$ duration $100 \mathrm{~Hz}$ conditioning trains with an inter-train interval of $10 \mathrm{~s}$. We then returned to stimulating synaptic responses every $30 \mathrm{~s}$. As shown in both the example experiment (Figure 2A) and the pooled data set (Figure 2C), in WT animals the EPSP amplitude was approximately doubled in size in the 2 minutes immediately following application of the conditioning stimulus $(1.97 \pm 0.1$ fold increase, $\mathrm{n}=7)$. This post-tetanic potentiation rapidly decayed to a significant steady level of LTP which averaged $26 \pm 5 \%$ greater than baseline 26-30 minutes after application of the conditioning stimulus $(\mathrm{P}<0.01, \mathrm{n}=7$, paired t-test). In slices from Tg2576 mice (Figure $2 \mathrm{~B}$ and $2 \mathrm{C}$ ) the posttetanic potentiation observed immediately after the

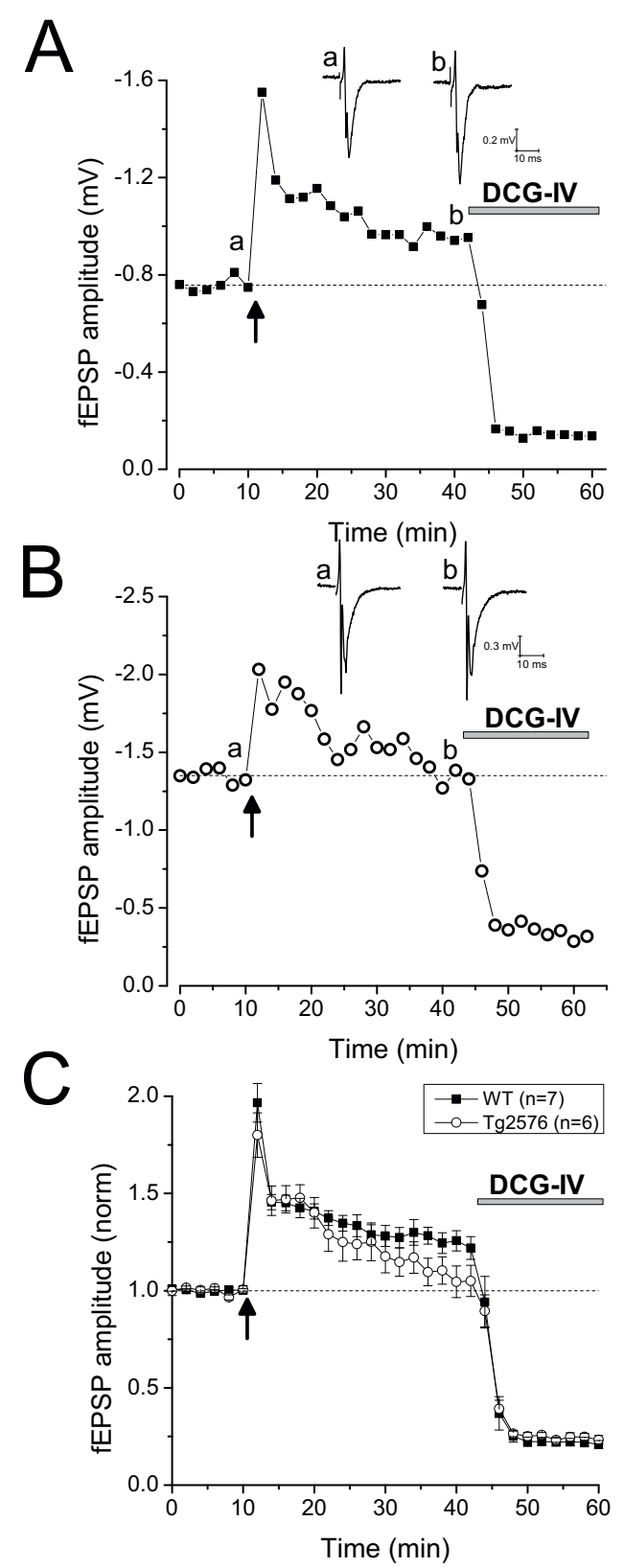

Figure 2 Loss of NMDA-receptor independent LTP in the mossy fibre pathway of Tg2576 mice. A) An example LTP experiment in a 25 month old WT mouse. After a 10 minute period of stable baseline EPSPs in response to low frequency stimulation $(0.05 \mathrm{~Hz})$, a conditioning stimulus consisting of $3,1 \mathrm{~s}, 100 \mathrm{~Hz}$ train was applied at the time indicated by the arrow. Post conditioning low frequency stimulation-evoked EPSPs were followed for another 30 minutes after which time the synaptic depression produced by DCG-IV $(2 \mu \mathrm{M})$ application was determined. The inset show example traces recorded just prior to the conditioning stimulus and just prior to DCG-IV application. B) An example recording similar to that in (A) but from a Tg2576 mouse. C) Pooled LTP data from 7 WT and 6 Tg2576 slices (taken from 4 and 5 animals, respectively). Note the LTP in slices prepared from Tg2576 mice does not persist. DCG-IV consistently depressed the synaptic responses by $\sim 80 \%$ in both groups. 
conditioning stimulus was $1.80 \pm 0.11$ fold $(\mathrm{n}=6)$, not significantly different from that in WT mice $(\mathrm{P}>0.29$, unpaired t-test). However, after 30 minutes the EPSP amplitude had decayed to $1.05 \pm 0.08$ fold of baseline. This level was not significantly different from the EPSP amplitude prior to applying the conditioning stimulus ( $\mathrm{P}$ $>0.44$, paired t-test). Furthermore, comparison of the extent of LTP between Tg2576 and WT mice revealed a significant difference $(\mathrm{P}<0.04$, unpaired $\mathrm{t}$-test).

To demonstrate that we were indeed studying MFPmediated synaptic transmission the group II mGluR agonist DCG-IV was applied to every slice 30 minutes after the application of the $100 \mathrm{~Hz}$ conditioning stimuli [17]. As reported in younger animals, this agent produced a robust depression of the EPSP in both WT and Tg2576 mice (Figures 2A, B and 2C).

\section{Discussion}

This manuscript provides a first description of alterations to MF synaptic function in a mouse model of ADrelated $A \beta$ accumulation. For this work we used a single cohort of Tg2576 mice which express the Swedish mutation of APP under the control of a prion protein promoter. These animals are an early example of the now numerous mouse lines genetically engineered to overproduce $A \beta$ in an age-dependent fashion. Such animals are commonly used to experimentally model certain aspects of AD pathology [4] and are also widely employed in the quest to develop $A \beta$-lowering agents as potential therapies for AD.

Tg2576 mice were first produced some 15 years ago [15] and to date have been the most widely studied $A \beta$ overproducing mice. Tg2576 mice were the first to be used to demonstrate alterations to hippocampal synaptic function in both the PP input to the dentate gyrus and the SCCP input to area CA1 [18]. Numerous related studies have also been performed on more recently generated $A \beta$-overproducing lines, many of which exhibit a more aggressive and rapidly arising amyloidopathy [5]. These have included investigations of synaptic function and plasticity in both the PP and SCCP, which when compared reveal rather mixed and often conflicting findings [5].

Despite the numerous investigations in the PP and SCCP, to our knowledge there have been no previous studies of MF function in any $A \beta$ overproducing mouse lines; although there has been a single study of the MFP in $\beta$-secretase knockout mice which are unable to produce $A \beta$. These $\beta$-secretase null animals exhibit enhanced MF paired-pulse facilitation and loss of LTP [19]. Here we describe how mossy fibre function is altered in Tg2576 mice. Our study was performed with mice aged $\sim 2$ years; thus, in addition to determining the consequences of an increased $A \beta$ load at these advanced ages, our data also provide a first insight in of MF function in very aged WT mice.

2 year old WT mice were found to have extracellularly recorded synaptic responses that outwardly resemble the distinctive transient waveform of MF fEPSPs in younger animals. Our recordings also indicate that the presence of quite substantial, long-lasting (i.e. $>1$ s) paired-pulse facilitation (Figure 2B) combined with strong $1 \mathrm{~Hz}$ frequency facilitation (Figure 2C), both hallmarks of MFCA3-PC transmission in younger animals [20], appear to persist into old age.

It has been previously shown that the extent of pairedpulse and frequency facilitation in the MFP of mice declines over the first 9 post natal weeks [21]. Although it is not easy to directly compare across laboratories due to differences in experimental methods (e.g. recording temperature, divalent ion concentration, species and strain employed), our data certainly indicate that there is not much further decline in the extent of MF short term plasticity as animals age from 9 weeks to 2 years, a supposition that is supported by other recent work on the MFP of adult rodents in our laboratory. It is also important to note that significant NMDA receptor-independent MF-LTP can also be evoked in aged WT mice; in our recordings it was seen in every slice, varying in amplitude from $7 \%$ to $45 \%$ of baseline amplitude, and was always preceded by a rapidly decaying post-tetanic potentiation.

In studies of the MFP it is standard practice to apply DCG-IV at the end of recordings. This is used to demonstrate that synaptic responses arise largely from stimulation of MFs, rather than recruitment of other glutamatergic pathways, notably the associational-commissural fibres, that can also generate glutamatergic synaptic responses in area CA3 [17]. We were reassured to see our synaptic responses were consistently depressed by around $80 \%$ by this group II mGluR agonist (Figure 2).

In contrast with studies of the SCCP in A $\beta$ overproducing mice, including those performed in our laboratory [22-25], input-output properties of MF responses were not dependent on genotype (Figure 1A). In contrast, both short term synaptic plasticity and LTP in the MFP were modified in the $\mathrm{A} \beta$ peptide over-producing mice. The PPF deficit observed in Tg2576 was subtle (Figure 1B); for example, with two stimuli applied with a $1 \mathrm{~s}$ inter-stimulus interval, PPF was $\sim 8 \%$ smaller in Tg2576 mice. Although small, the PPF reduction in the $\mathrm{A} \beta$-overproducing animals mirrors and indeed may be related to the enhanced PPF seen in BACE knockout mice, animals which can not produce $A \beta$ [19]. Together these findings suggest $A \beta$ may exert a tonic control of short term plasticity in wild-type non-transgenic mice. A similar conclusion was recently made from work 
largely based on hippocampal cultures [26]. Changes in PPF usually reflect an altered probability of release. The relatively minor (5-10\%) decreases to PPF observed in the MFP of Tg2576 mice suggest that any underlying increases in release probability at stimulus-naïve synapses would also be small. We believe this explains why we do not see significantly enhanced input-output curves in the transgenic mice, especially in light of the considerable slice to slice variations in input-output relationships

Genotype-related differences in short-term MF plasticity were more markedly apparent in experiments in which 20 pulse, $1 \mathrm{~Hz}$ stimulus trains were used to induce frequency facilitation. The rate with which EPSPs in slices from Tg2576 mice increased was similar to that seen in WT mice (Figure 2D), but responses only tripled in size compared with a 4.5 fold change seen in WT animals. Interestingly, the changes in MF frequency facilitation in Tg2576 mice are very similar to those we have recently seen in TC1 mice at $\sim 6$ months of age. TC1 mice are a transchromosomic model of Down's syndrome, which like Tg2576 mice, exhibit deficits in hippocampal learning [27]. Unlike Tg2576 mice (Figure 2), however, TC1 mice do not have a parallel deficit in MF LTP (manuscript in preparation).

The marked frequency facilitation of normal MF responses is proposed endow this pathway with important circuit properties not seen in other hippocampal pathways. Thus, if individual DG cells fire repeatedly, the synaptic responses they generate in CA3 pyramids rapidly grow, such that one DG neurone can drive postsynaptic firing in CA3 cells [14]. Indeed, these features have lead to these synaptic connections being dubbed as "detonator synapses" [7]. Thus, the substantial loss of frequency facilitation in Tg2576 animals could have significant implications for how repetitive activity in DGGCs is able to drive synaptic responses and subsequent action potential firing in post-synaptic CA3-PCs. In future, it will be interesting to examine the cellular basis for the depression of MF frequency facilitation. For example, an interesting potential avenue of investigation would be to examine if the presynaptic kainate receptormediated component of frequency facilitation [28] has been lost in MFs of Tg2576 mice.

It is also worth noting that both FF and PPF in MF are thought to reflect the actions of residual presynaptic $\mathrm{Ca}^{2+}$. Despite this common source, changes to the frequency facilitation appear to be more profound in Tg2576 mice. There are a number of potential causes for this that could be addressed in the future. For example, the difference between PPF and FF could arise from genotype-related differences in the dynamics or capacity of processes controlling presynaptic $\mathrm{Ca}^{2+}$ homeostasis, or from differences in the size of the readily releasable vesicle pool.
In addition to changes to short-term plasticity, the ability of MF to exhibit activity-dependent LTP was compromised in 2 year old Tg2576 mice. Indeed, 30 mins after an induction protocol that induced LTP in all WT slices examined, there was no significant LTP in the Tg2576 group. The effects of transgenic A $\beta$ overproduction on NMDA receptor-dependent LTP in the PP and SCCP remain somewhat controversial, with some laboratories seeing deficits and others, including ourselves, seeing no differences (reviewed by [5]). To some degree this research question is not aided by different groups using different transgenic lines, of different ages and different protocols. We believe that this is the first study of LTP in the MFP of an A $\beta$ overproducing mouse, it will be interesting to see if other investigators make similar findings in future. Furthermore, a mechanistically different, NMDA receptor-dependent form of LTP has recently been described in the MFP of 2-4 week old rodents. This plasticity is postsynapticallymediated and specifically involves potentiation of NMDA receptor-mediated synaptic responses [29,30]. Assuming this non-classical form MF LTP persists into adulthood, it would be informative to investigate if and how it is affected by $\mathrm{A} \beta$ accumulation.

In combination with alterations to other hippocampal circuits, the altered long- and short-term synaptic plasticity in the MF inputs to CA3-PC presumably contribute to altered hippocampal function that ultimately leads to learning and memory deficits in $A \beta$-overproducing mice, and by extension human AD sufferers. To fully understand all the consequences of prolonged $A \beta$ accumulation for information transfer between DG and area CA3 it will also be important to investigate function and plasticity in MF connections with GABAergic interneurones. It will be particularly fascinating to examine the interneurones in stratum lucidum which are innervated by filopodial extensions from the large MF boutons that innervate pyramidal cells. Interestingly, at least in young animals, these connections often exhibit paired pulse depression and high frequency stimulation-induced long-term depression [31].

\section{Conclusions}

We have provided the first evidence that MF transmission is disturbed in an $\mathrm{A} \beta$-overproducing transgenic mouse line. This adds to the catalogue of neurophysiological deficits described in such models of AD-associated amyloidopathy, and uncovers the need for further AD-related investigations of this neurophysiologically unique component of the trisynaptic loop.

\section{Abbreviations}

A $\beta$ : amyloid beta peptide; AD: Alzheimer's disease; APP: amyloid precursor protein; DG: dentate gyrus; LTP: long-term potentiation; MF: mossy fibre(s); 
MFP: mossy fibre projection; PP: perforant pathway; PPF: paired pulse facilitation; SCCP: Schaffer collateral commissural pathway; WT: wild-type.

\section{Acknowledgements}

The transgenic mice used in this study were provided by Pfizer who also provided financial support for consumable costs. JW is a PhD student funded by a Medical Research Council Industrial Collaborative Studentship awarded to AR and MJ and co-funded by Pfizer.

\section{Author details}

'School of Physiology and Pharmacology, University of Bristol, University Walk, Bristol, BS8 1TD, UK. ²Pfizer Applied Neurophysiology Group, University of Bristol, University Walk, Bristol, BS8 1TD, UK.

\section{Authors' contributions}

The experimental work was largely performed by JW with training and experimental and analytical support from JB. AR and MJ co-supervise JW and were involved in the planning of these studies, obtaining research funding and preparation of the manuscript. All authors read and approved the final manuscript

\section{Competing interests}

The authors declare that they have no competing interests

Received: 16 September 2010 Accepted: 1 November 2010 Published: 1 November 2010

\section{References}

1. Hardy JA, Higgins GA: Alzheimer's disease: the amyloid cascade hypothesis. Science 1992, 256:184-185.

2. Pimplikar SW: Reassessing the amyloid cascade hypothesis of Alzheimer's disease. Int J Biochem Cell Biol 2009, 41:1261-1268.

3. Chow WW, Mattson MP, Wong PC, Gleichmann M: An Overview of APP Processing Enzymes and Products. Neuromolecular Med 2009, 12:1-12.

4. Morrissette DA, Parachikova A, Green KN, LaFerla FM: Relevance of transgenic mouse models to human Alzheimer disease. J Biol Chem 2009, 284:6033-6037.

5. Randall AD, Witton J, Booth C, Hynes-Allen A, Brown JT: The functional neurophysiology of the amyloid precursor protein (APP) processing pathway. Neuropharmacol 2010, 59:243-267.

6. Henze DA, Urban NN, Barrionuevo G: The multifarious hippocampal mossy fiber pathway: a review. Neuroscience 2000, 98:407-427.

7. Urban NN, Henze DA, Barrionuevo G: Revisiting the role of the hippocampal mossy fiber synapse. Hippocampus 2001, 11:408-417.

8. Nicoll RA, Schmitz D: Synaptic plasticity at hippocampal mossy fibre synapses. Nat Rev Neurosci 2005, 6:863-876.

9. Lawrence JJ, McBain CJ: Interneuron diversity series: containing the detonation-feedforward inhibition in the CA3 hippocampus. Trends Neurosci 2003, 26:631-640.

10. Bischofberger J, Engel D, Frotscher M, Jonas P: Timing and efficacy of transmitter release at mossy fiber synapses in the hippocampal network. Pflugers Arch 2006, 453:361-372.

11. Cerasti $E$, Treves A: How informative are spatial CA3 representations established by the dentate gyrus? PLoS Comput Biol 2010, 6:e1000759.

12. Lassalle JM, Bataille T, Halley H: Reversible inactivation of the hippocampal mossy fiber synapses in mice impairs spatial learning, but neither consolidation nor memory retrieval, in the Morris navigation task. Neurobiol Learn Mem 2000, 73:243-257.

13. Lee I, Kesner RP: Encoding versus retrieval of spatial memory: double dissociation between the dentate gyrus and the perforant path inputs into CA3 in the dorsal hippocampus. Hippocampus 2004, 14:66-76.

14. Treves A, Tashiro A, Witter ME, Moser El: What is the mammalian dentate gyrus good for? Neuroscience 2008, 154:1155-1172.

15. Hsiao K, Chapman $P$, Nilsen $S$, Eckman $C$, Harigaya $Y$, Younkin S, Yang F, Cole G: Correlative memory deficits, Abeta elevation, and amyloid plaques in transgenic mice. Science 1996, 274:99-102.

16. Mistry R, Dennis S, Frerking M, Mellor JR: Dentate gyrus granule cell firing patterns can induce mossy fiber long-term potentiation in vitro. Hippocampus 2010.
17. Kamiya $H$, Shinozaki $H$, Yamamoto C: Activation of metabotropic glutamate receptor type $2 / 3$ suppresses transmission at rat hippocampal mossy fibre synapses. J Physiol 1996, 493:447-455.

18. Chapman PF, White GL, Jones MW, Cooper-Blacketer D, Marshall VJ, Irizarry M, Younkin L, Good MA, Bliss TV, Hyman BT, et al: Impaired synaptic plasticity and learning in aged amyloid precursor protein transgenic mice. Nat Neurosci 1999, 2:271-276.

19. Wang H, Song L, Laird F, Wong PC, Lee HK: BACE1 knock-outs display deficits in activity-dependent potentiation of synaptic transmission at mossy fiber to CA3 synapses in the hippocampus. J Neurosci 2008, 28:8677-8681.

20. Salin PA, Scanziani M, Malenka RC, Nicoll RA: Distinct short-term plasticity at two excitatory synapses in the hippocampus. Proc Natl Acad Sci USA 1996, 93:13304-13309.

21. Mori-Kawakami F, Kobayashi K, Takahashi T: Developmental decrease in synaptic facilitation at the mouse hippocampal mossy fibre synapse. $J$ Physiol 2003, 553:37-48.

22. Brown JT, Richardson JC, Collingridge GL, Randall AD, Davies CH: Synaptic transmission and synchronous activity is disrupted in hippocampal slices taken from aged TAS10 mice. Hippocampus 2005, 15:110-117.

23. Fitzjohn SM, Morton RA, Kuenzi F, Rosahl TW, Shearman M, Lewis $H$ Smith D, Reynolds DS, Davies CH, Collingridge GL, Seabrook GR: Agerelated impairment of synaptic transmission but normal long-term potentiation in transgenic mice that overexpress the human APP695SWE mutant form of amyloid precursor protein. J Neurosci 2001, 21:4691-4698.

24. Hsia AY, Masliah E, McConlogue L, Yu GQ, Tatsuno G, Hu K, Kholodenko D, Malenka RC, Nicoll RA, Mucke L: Plaque-independent disruption of neural circuits in Alzheimer's disease mouse models. Proc Natl Acad Sci USA 1999, 96:3228-3233.

25. Fitzjohn SM, Kuenzi F, Morton RA, Rosahl TW, Lewis H, Smith D, Seabrook GR, Collingridge GL: A study of long-term potentiation in transgenic mice over-expressing mutant forms of both amyloid precursor protein and presenilin-1. Mol Brain 2010, 3:21.

26. Abramov E, Dolev I, Fogel H, Ciccotosto GD, Ruff E, Slutsky I: Amyloid-beta as a positive endogenous regulator of release probability at hippocampal synapses. Nat Neurosci 2009, 12:1567-1576.

27. O'Doherty A, Ruf S, Mulligan C, Hildreth V, Errington ML, Cooke S, Sesay A Modino S, Vanes L, Hernandez D, et al: An aneuploid mouse strain carrying human chromosome 21 with Down syndrome phenotypes. Science 2005, 309:2033-2037.

28. Schmitz D, Mellor J, Frerking M, Nicoll RA: Presynaptic kainate receptors at hippocampal mossy fiber synapses. Proc Natl Acad Sci USA 2001, 98:11003-11008.

29. Kwon HB, Castillo PE: Long-term potentiation selectively expressed by NMDA receptors at hippocampal mossy fiber synapses. Neuron 2008, 57:108-120.

30. Rebola N, Lujan R, Cunha RA, Mulle C: Adenosine A2A receptors are essential for long-term potentiation of NMDA-EPSCs at hippocampal mossy fiber synapses. Neuron 2008, 57:121-134.

31. Pelkey KA, Topolnik L, Lacaille JC, McBain CJ: Compartmentalized $\mathrm{Ca}(2+)$ channel regulation at divergent mossy-fiber release sites underlies target cell-dependent plasticity. Neuron 2006, 52:497-510.

doi:10.1186/1756-6606-3-32

Cite this article as: Witton et al:: Altered synaptic plasticity in the mossy fibre pathway of transgenic mice expressing mutant amyloid precursor protein. Molecular Brain 2010 3:32. 